\title{
Estudio de campo sobre la eficacia de la aplicación de dos modalidades clásicas de triple terapia antirretroviral
}

\author{
J. M. EIROS BOUZA, M. ORTEGA LAFONT, R. ORTIZ DE LEJARAZU, \\ P. BACHILLER LUQUE, D. A. DE LUIS ROMÁN \\ Departamentos de Microbiología y Medicina Interna. Hospital Clínico Universitario y \\ Hospital Universitario Río Hortega. Valladolid
}

\section{RESUMEN}

Fundamento: Comparar la evolución temporal de los parámetros de carga viral (CV) y CD4 en dos cohortes de pacientes VIH tratados con dos regímenes clásicos de terapia antirretroviral.

Métodos: Estudio retrospectivo, observacional, y descriptivo del porcentaje de pacientes que alcanzaron cargas virales no detectables y el tiempo que tardaron en lograrlo. Las cohortes fueron: 91 pacientes tratados con zidovudina, lamivudina e indinavir (cohorte A) versus 80 pacientes tratados con estavudina, didanosina e indinavir (cohorte B).

Resultados: La evolución de los pacientes fue similar en cuanto al porcentaje de los mismos que alcanzaron CV "no detectables" (75,8\% en la cohorte A y 73,8\% en la cohorte B) a lo largo del tiempo de seguimiento (cuatro años). El tiempo medio transcurrido hasta alcanzar el referido "éxito" fue diferente, 209 días (IC 95\%: 175-243 días) en el caso del régimen A y 330 días (IC 95\%: 263-396 días) para el régimen B. El estado inmunológico en el momento de su primera CV "no detectable" de los pacientes que recibían Zidovudina, Lamivudina e Indinavir se encontraba significativamente más conservado que en el otro grupo $(83,1$ frente a $65,4 \%$ para cifras de linfocitos $\mathrm{CD} 4 / \mathrm{mm} 3$ superiores a 200 , respectivamente; $\mathrm{p}=0,032$ ).

Conclusiones: En nuestro estudio si bien la eficiencia de ambas combinaciones terapéuticas resultó equiparable en cuanto a la similitud del porcentaje de individuos que alcanzaron viremias "no detectables" a lo largo del tiempo de seguimiento, aquéllos tratados con Zidovudina, Lamivudina e Indinavir lo lograron antes.

PALABRAS CLAVE: Carga viral. CD4. Antirretrovirales.
OBSERVATIONAL STUDY OVER THE EFFICACY OF THE APPLICATION OF TWO CLASSICAL REGIMENS OF TRIPLE ANTIRETROVIRAL THERAPY

ABSTRACT

Background: To compare the temporal evolution of viral load and CD4 parameters in two cohorts of HIV infected patients enrolled in classical triple antiretroviral regimens.

Methods: Retrospective, observational, descriptive study of the proportions of patients reaching undetectable levels of viral load $(V L)$ as well as the time necessary to get it. The two cohorts were as follows: 91 HIV patients on triple therapy with zidovudine plus lamivudine and indinavir (cohort A) versus 80 HIV patients with Stavudine plus Didanosine and Indinavir (cohort $B$ ).

Results: The evolution of the patients in terms of percentages who reach undetectable VL was similar in the two therapeutic cohorts $75.8 \%$ for cohort A vs $73.8 \%$ for cohort B) along the duration of the study (four years). However, the mean time period needed to reach undetectable $V L$ was different, 209 days (IC 95\% 175-243 days) for patients in zidovudine plus lamivudine and indinavir and 330 days (IC 95\% 263-396 days) for stavudine plus didanosine and indinavir regimen. The immunological status observed in the patients when reaching his first undetectable VL was significantly different. The proportion of patients with CD4 cells counts $>200 / \mathrm{mm} 3$ in cohort A was $83.1 \%$ while for patients from cohort $B$ was $65.4 \%(p=0.032)$.

Conclusions: This observational study from clinical settings seems demonstrate similar efficacy to reach undetectable VL with both classical triple antiretroviral therapies evaluated but a shorter delay of time to reach that virological situation for zidovudine plus lamivudine and indinavir regimen is reported.

KEY WORDS: Viral load. CD4. Antiretroviral treatment.

Eiros Bouza JM, Ortega Lafont M, Ortiz de Lejarazu R, Bachiller Luque P, de Luis Román DA. Estudio de campo sobre la eficacia de la aplicación de dos modalidades clásicas de triple terapia antirretroviral. An Med Interna (Madrid) 2003; 20: 239-242.

\section{INTRODUCCIÓN}

La utilización de terapias combinadas de varios fármacos antirretrovirales ha sido clave para conseguir remisiones importantes de la replicación del virus de la inmunodeficiencia humana (VIH) y para minimizar la aparición de resisten- cias frente al mismo $(1,2)$. La incorporación desde finales de 1996 de los denominados regímenes de alta eficacia ha condicionado la historia natural de la infección, reduciendo la mortalidad hasta en un $90 \%(3,4)$. Los innegables avances en este ámbito se han visto condicionados por la incapacidad del paciente para mantener una óptima adherencia $(5,6)$ y por la

Trabajo aceptado: 16 de enero de 2003

Correspondencia: JM Eiros Bouza. Facultad de Medicina. Sexta planta. Avda. Ramón y Cajal, 7.47005 Valladolid. e-mail: eiros @med.uva.es 


\section{DISCUSIÓN}

En el ámbito de la terapia antirretroviral existe un importantísimo número de publicaciones cuyo crecimiento en el último quinquenio ha sido exponencial, sin embargo son más escasos los estudios observacionales de la práctica clínica asistencial. Los grandes estudios que empleaban criterios de valoración tanto clínicos, como de supervivencia tales como Concorde (10), ACTG-175 (11), NUCA (12), NUCB 3001/ NUCB 3002 (13), CAESAR (14) o Delta (15), aunque condujeron a conclusiones espectaculares, pronto se vieron superados merced a la aparición de técnicas que posibilitan la detección de la viremia (16). Nuestra contribución al comentar los hallazgos del presente estudio no es otra que la de poner de manifiesto la realidad en la prescripción de la terapia recogida desde la óptica de un centro de referencia que determina CV desde el inicio de la implantación de este parámetro a la oferta diagnóstica de un hospital de tercer nivel.

La serie aquí descrita recoge individuos infectados por VIH que fueron evaluados en el marco temporal de la plena implantación de la triple terapia, pero en el que se ofertaban una gran diversidad de opciones terapéuticas. El análisis se circunscribió a una modalidad que incluía zidovudina, lamivudina e Indinavir y a otra formada por didanosina, Estavudina e Indinavir. La oportunidad de dirigir nuestro análisis a estos dos subgrupos se justificó por el hecho de que ambos eran homogéneos en lo relativo a diez variables deliberadamente controladas tal y como se ha expuesto en el apartado de pacientes y métodos.

La eficiencia de ambas combinaciones terapéuticas resultó equiparable en cuanto a la similitud del porcentaje de individuos que alcanzaron viremias "no detectables" a lo largo del tiempo de seguimiento; si bien el tiempo medio transcurrido hasta lograr este evento fue inferior en la primera modalidad (ZDV, 3TC, IDV) frente a la segunda (ddI, D4T, IDV) tal y como resulta evidente al comparar los intervalos de confianza del 95\% de las medias del tiempo (175-243 días frente a 263396 días). En el mismo sentido se inscribe el que el estado inmunológico de los pacientes del primer grupo se encontrara significativamente más conservado en el momento del éxito que en el segundo grupo (linfocitos CD4 $>200 / \mathrm{mm}^{3}$ en el $83,1 \%$ vs $65,4 \%$; $\mathrm{p}=0,032$ ).

Una reflexión adicional es la relativa a que para una pequeña proporción de pacientes con valores muy similares se documentó el fracaso en ambos grupos $(8.8 \%$ y $11.3 \%)$. A pesar de que el tiempo en el que ocurrió este evento fue más prolongado en el primer grupo (469 días) que en el segundo (393 días), la amplitud de los intervalos de confianza matiza la significación del hallazgo.

Nuestro estudio no obedece a un diseño para la evaluación de eficiencia de terapias y en consecuencia no se han controlado otras variables que influyen en el desarrollo del éxito terapéutico como adherencia (17), y las relativas a la farmacocinética (18), pero resulta útil comprobar que en la práctica asistencial las modificaciones de la viremia están ligadas a la modalidad terapéutica prescrita. En el momento actual el aumento de la oferta terapeútica (19-23) amplía sustancialmente el campo de trabajo a la hora de evaluar su impacto clínico. Ello redundará sin duda en un avance sustancial a la hora de individualizar el tratamiento antirretroviral (24).

\section{Bibliografía}

1. Hanna GJ, D' Aquila RT. Clinical use of genotypic and phenotypic drug resistance testing to monitor antiretroviral chemotherapy. Clin Infect Dis 2001; 32: 774-782

2. US Dept of Health and Human Services. Guidelines for the use of antiretroviral agents in HIV-infected adults and adolescens. Washington, DC: US Dept of Health and Human Services, 2002. Accesible en: http://www.hivatis.org/trtgdlns.html.

3. Palella FJ, Delaney KM, Moorman AC, Loveless MO, Fuhrer J, Satten GA et al. Declining morbility and mortality among patients with advanced Human Immunodeficiency Virus infection. N Engl J Med 1998; 338: 853-860.

4. Kaufmann D, Pantaleo G, Sudre P, Telenti A. CD4-cell count in HIV-1 infected individuals remaining viraemic with highly antiretroviral therapy (HAART). Swiss Cohort Study. Lancet 1998; 351: 723-724.

5. Paterson DL, Swindells S, Mohr J, Brester M, Vergis EN, Squier C et al. Adherence to protease inhibitor therapy and outcomes in patients with HIV infection. Ann Intern Med 2000; 133: 21-30.

6. Markowitz M. Resistence, fitness, adherence and potency: mapping the paths to virologic failure. JAMA 2000; 283: 250-251.

7. Torre D, Tambini R. Antiretroviral drug resistance testing in patients with HIV-1 infection: A meta-analysis study. HIV Clin Trials 2002; 3: $1-8$.

8. Arrizabalaga J, Alcamí J, Dalmau D, Delgado R, Miró JM; Soriano V. Herramientas de laboratorio para individualizar el tratamiento: resistencias y niveles de fármacos. Enferm Infecc Microbiol Clin 2002; 20 (Supl 2): 35-47.

9. Labayru C, Hernández B, Eiros JM, Ortega M, Castrodeza J, Ortiz de Lejarazu R, Rodríguez Torres A. Resistencias genotípicas del virus de la inmunodeficiencia humana en pacientes con fracaso virológico. Med Clin (Barc) 2002; 119: 201-205.

10. Aboulker JP, Swart AM. Preliminary analysis of the Concorde trial. Coordinating commitee. Lancet 1993; 341: 889-890.

11. Hammer SM, Katzenstein DA, Hughes MD, Gundacker H, Schooley RT, Haubrinch RH et al. A trial comparing nucleoside monotherapy with combination therapy in HIV-infected adults with CD4 cell counts from 200 to 500 per cubic millimeter. N Engl J Med 1996; 355: 10811090 .

12. Katlama C, Ingrand D, Loveday C, Clumeck N, Mallolas J, Staszewski $\mathrm{S}$ et al. Safety and efficaccy of lamivudine-zidovudine combination therapy in antiretroviral-naive patients. JAMA 1996; 276:118-125.

13. Katlama C, Ingrad D, Loveday C, Clumeck N, Mallolas J, Staszewski $\mathrm{S}$ et al. Safety and efficaccy of lamivudine-zidovudine combination therapy in zidovudine-experienced patients. A randomized controlled comparison with zidovudine monotherapy. JAMA 1996; 276:111117.

14. Dore GJ, Cooper DA, Barrett C, Goh LE, Thakrara B, Atkins M. Dual efficacy of lamivudine treatment in human immunodeficiency/hepatitis $B$ virus-coinfected persons in a randomized, controlled study (CAESAR). The CAESAR Coordinating Commitee. J Infect Dis 1999; 180: 607-613.

15. Hirsch MS, Yeni P. A bend in the road-implications of ACTG 175 and Delta trials. Antivir Ther 1996; 1: 6-8.

16. Clementi M, Menzo S, Bagnarelli P, Valenza, Paolucci S, Sampaolesi $\mathrm{R}$ et al. Clinical use of quantitative molecular methods in studing Human Immunodeficiency Virus Type 1 infection. Clin Microbiol Rev 1996; 9: 135-147. 